\title{
Correlation Between Pain, Fear of Falling and Disability in Low Back Pain
}

\author{
Sadhana Verma, MPT $^{1}$, Bharat Prakash Pal, BPT ${ }^{2}$ \\ ${ }^{1}$ Department of Physiotherapy, Lala Lajpat Rai Hospital, Kanpur; \\ ${ }^{2}$ Department of Physiotherapy, Saai College of Medical Science and Technology, Kanpur, India
}

Objective To ascertain if there is a correlation between low back pain (LBP), fear of falling, and disability so that the patients with LBP are aware of the fact that other problems may occur with LBP. Hence, steps can be taken for decreasing the fear of falling and disability in order to improve the condition of patients.

Methods A sample size of 100 patients with low back pain, with a range of ages from 40 to 73 years, participated in the study. The Falls Efficacy Scale was used to assess the fear of falling and the Oswestry Disability Index was used to assess the disability and pain in LBP individuals.

Results The Pearson correlation analysis signifies the relationship between pain, fear of falling, and disability in LBP.

Conclusion First, LBP increases the fear of falling. Second, LBP can result in a person becoming disabled. Third, the fear of falling and disability are correlated with each other.

Keywords Low back pain, Fear, Fall, Morbidity

\section{INTRODUCTION}

Low back pain (LBP) is a common health condition that affects most adults at some point during their lifetime. For most patients in primary care, the source of symptoms cannot be specified and the patient is labeled with a diagnosis called non-specific LBP [1].

LBP is among the most important factors affecting

Received August 5, 2013; Accepted April 16, 2014

Corresponding author: Sadhana Verma

Department of Physiotherapy, Saai College of Medical Science and Technology, Chaubepur, Kanpur-208011, India

Tel: +91-2631667, Fax: +91-9793673384,E-mail: vermasadhana85@ yahoo.com

(c) This is an open-access article distributed under the terms of the Creative Commons Attribution Non-Commercial License (http://creativecommons. org/licenses/by-nc/4.0) which permits unrestricted noncommercial use, distribution, and reproduction in any medium, provided the original work is properly cited.

Copyright $\odot 2015$ by Korean Academy of Rehabilitation Medicine health status and functional capacity of elderly people, with a prevalence of $12 \%$ to $42 \%$ in subjects over 65 years of age. It is more common in older women than in men, and women are more likely to have LBP for prolonged periods. It was found out that severe back pain increases the likelihood of disability by 3 - to 4 -fold, whereas mild or moderate back pain is not associated with reduced functional activities of daily living, LBP is found to be related to 2 -fold greater difficulty in daily living activities [2]. A large percentage of LBP results in a high rate of morbidity. An important amount of labor lost because of this pain can be relieved by non-invasive conservative treatment.

Unfortunately, about 5\% of LBP becomes chronic. This pain continues to be the underlying cause of severe pain and functional disorder. Although there are many sources of pain in the low back region, intervertebral discs are one of the most important sources of LBP. It has been 
suggested that internal disc disruption (IDD) is the cause of $40 \%$ of chronic, persistent, LBP of unknown origin [3].

Falls are one of the major health problems among older adults. Consequently, deterioration of a condition leads to the loss of independence through decreased mobility and increased fear of falling. A decrease in mobility and independence are often serious enough to result in hospital or nursing home admission [4].

Progressive weakness and the increasing risk of falling and disability can be treated if it is properly assessed and treated thoroughly by a rehabilitation program. Thus, this study is an effort in finding out the relationship between low back pain, fear of falling, and disability.

\section{MATERIALS AND METHODS}

A sample size of 100 patients with LBP, with a range of ages from 40 to 70 years, participated in the study. The subjects were selected on the basis of inclusion and exclusion criteria.

The inclusion criteria were as follows: 1 ) patients with non-specific LBP for at least 6 weeks; 2) both male and female patients aged 40-70 years; 3 ) participants must have had at least one episode of LBP prior to the study; 4) normal lower extremity on neurological examination [3]; and 5) both falling and non-falling LBP patients were included in the study [5].

The exclusion criteria were as follows: 1) patients who had any previous spinal surgical application; 2) pregnant patients [3]; and 3) patients who had a herniated disc prolapse intervertebral disc [5].

\section{Protocol}

This was an experimental study in which there was a random selection that resulted in a sample of 100 LBP patients from Kanpur, India. All the participants were assessed by the aforementioned inclusion and exclusion criteria. Informed consent was given by all the participants was in the study. Thenceforth, there was an initial assessment, and a recording of the demographic details, the medical history and pain assessment, and fall history.

\section{Procedure}

The aim of study was explained to all patients. The Fall Efficacy Scale (FES) [6] was used for the assessment procedure for evaluating the fear of falling. The FES is a short, easy to administer tool that measures the level of concern about falling during social and physical activities, inside and outside the home, and whether or not the person actually does the activity. The level of concern is measured by the FES. The patients were asked 10 questions in which they were asked to state by score that ranged from 1 ('very confident') to 10 ('not confident at all'). Total scores ranged from 10 (best possible) to 100 (worst possible). Thus, lower scores indicated more confidence and higher scores indicated a lack of confidence and a greater fear of falling. The subjects were further categorized as low concern (score 16-19), moderate concern (score 20-27), high concern (score 28-64), and very high concern (a score greater than 64).

The Oswestry Disability Index (ODI) [7] was another questionnaire that was also used for the assessment of pain and disability in LBP patients. It is based on 10 questions, each having 6 alternatives that are scored from $0-5$, where zero denoted no problem at all and five denoted a serious problem. The sum after scoring was used as a percentage. If all ten sections were completed then doubling of the score was done for percentage calculation and if any section was excluded then the percentage was calculated by the formula:

$$
\% \text { of disability }=\frac{\text { Patient }^{\prime} \text { s score }}{\text { No. of sections completed } \times 5} \times 100
$$

The percentage of disability was then categorised as: minimum disability (0\%-20\%), moderate disability (21\%$40 \%)$, severe disability (41\%-60\%), crippling disability (61\%-80\%), and complete disability (81\%-100\%).

\section{RESULTS}

In patients with LBP the results of the descriptive analysis represented that there were 100 LBP subjects whose ages ranged between 40-73 years and consisted of $57 \mathrm{fe}$ males and 43 males.

The mean age of the subjects was $51.16 \pm 9.42$ years and the gender distribution was $1.43 \pm 0.50$.

The category and number of individuals was according to the percentage of disability category that was calculated through the ODI. The obtained results show that there is a good correlation between disability and the fear of falling, while through the statistical analysis, the severity of the disability was calculated and their frequency was 
estimated. Among these 100 LBP patients, $1 \%$ of the patients had minimum disability with a score between 0-20, $9 \%$ of the patients had a moderate disability with a score between $20-40,25 \%$ of the patients had a severe disability with a score $41-60,19 \%$ of LBP patients had a crippling disability with a score $61-80$, and $46 \%$ individuals were completely disabled with a score between 81-100. Thus the figures showed a distribution of disability or the extent of disability for the LBP individuals who participated in the study (Table 1).

The fear of falling frequency was calculated through the FES scale. It represented the distribution of fall because of LBP through the FES scale. None of subjects had a low concern score of 16-19. Eight percent patients had a moderate concern score of 20-27. Eighty-two percent patients had a high concern score of $28-64$, and 10 percent individuals had a very high concern score of greater than 64 (Table 2).

For a proper understanding we distributed the ODI into two components, an Oswestry Disability Index representing Pain (ODI-P) and an Oswestry Disability Index showing Disability (ODI-D). The Pearson correlation result for FES and ODI-D shows that the r-value is 0.48 and the pvalue is 0.00 , which signifies that FES and ODI-D are cor-

Table 1. Category of disability and percentage of individuals affected, as calculated by the Oswestry Disability Index

\begin{tabular}{|lc}
\hline \multicolumn{1}{|c}{ Category } & $\begin{array}{c}\text { \% of individuals } \\
\text { affected }\end{array}$ \\
\hline Minimum disability $(0 \%-20 \%)$ & 1 \\
\hline Moderate disability $(20 \%-40 \%)$ & 9 \\
\hline Severe disability $(41 \%-60 \%)$ & 25 \\
Crippling disability $(61 \%-80 \%)$ & 19 \\
Complete disability $(81 \%-100 \%)$ & 46 \\
\hline Total & 100 \\
\hline
\end{tabular}

Table 2. Fear of falling category and percentage of individuals according to the Fall Efficacy Scale

\begin{tabular}{|lc}
\hline \multicolumn{1}{c}{ Category } & $\begin{array}{c}\text { \% of individuals } \\
\text { affected }\end{array}$ \\
\hline Low concern (16-19 score) & 0 \\
\hline Moderate concern (20-27 score) & 8 \\
\hline High concern (28-64 score) & 82 \\
Very high concern (>64 score) & 10 \\
\hline Total & 100 \\
\hline
\end{tabular}

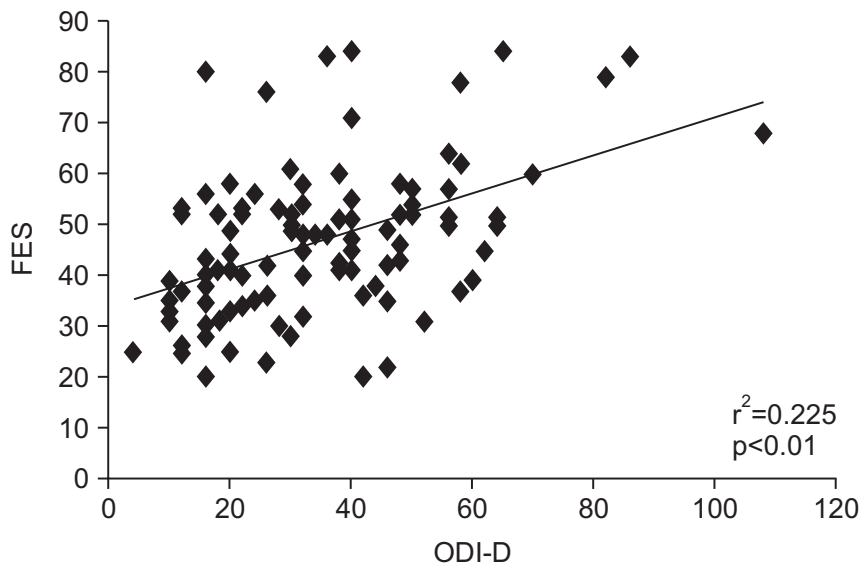

Fig. 1. Correlation between the Oswestry Disability Index showing Disability (ODI-D) and the Fall Efficacy Scale (FES).

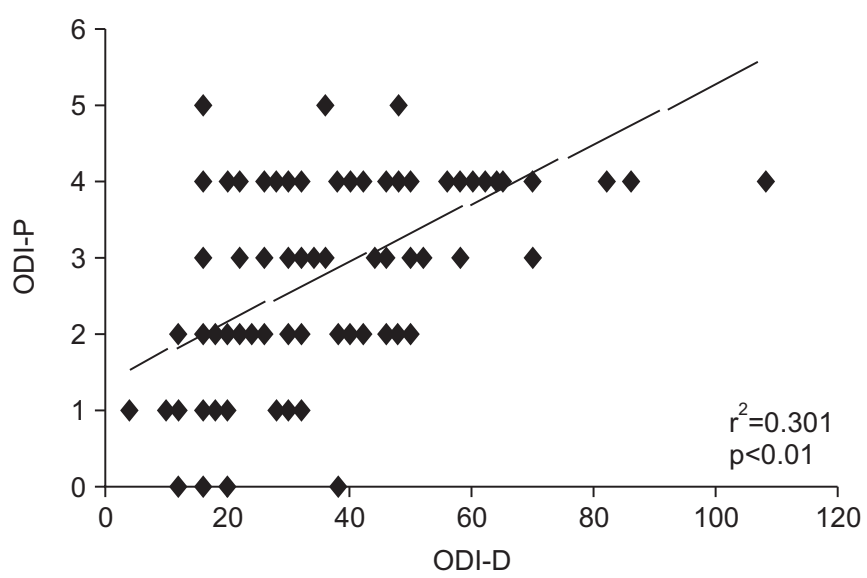

Fig. 2. Correlation between the Oswestry Disability Index representing Pain (ODI-P) and the Oswestry Disability Index showing Disability (ODI-D).

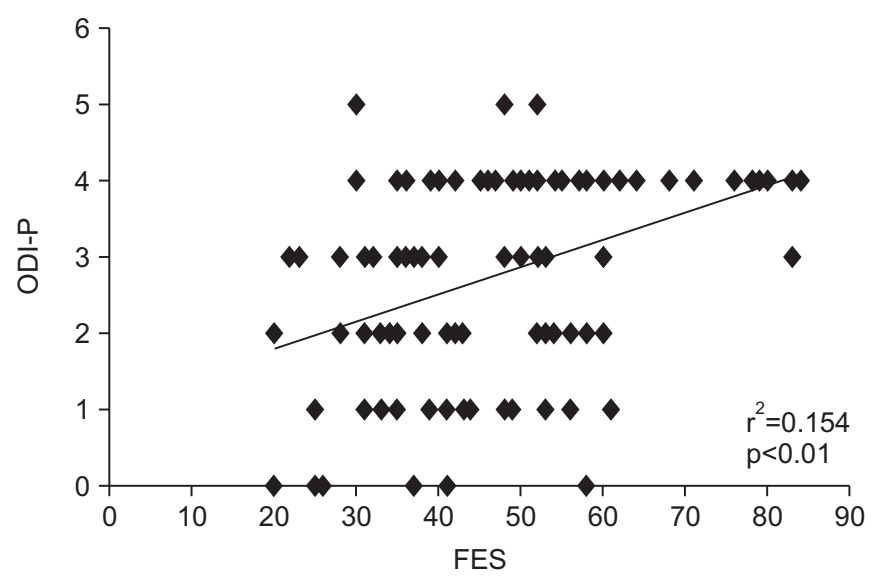

Fig. 3. Correlation between the Oswestry Disability Index representing Pain (ODI-P) and the Fall Efficacy Scale (FES). 
related to each other (Fig. 1).

While the correlation result for ODI-P and ODI-D represented that the r-value is 0.55 and the $\mathrm{p}$-value is $<0.01$. This shows that both ODI-D and ODI-P are related to each other. Furthermore, this shows that with pain, disability increases, and as the person becomes disabled, a greater stress on the muscles causes more and more pain (Fig. 2).

The correlation result between ODI-P and FES showed that the r-value is 0.39 and the p-value is $<0.01$, which is a significant value. A combined result between the three, that is, between ODI-D, ODI-P, and FES, show that all are related to each other (Fig. 3).

The correlation result for the age and ODI-P showed the r-value as -0.10 and the p-value as 0.33 , which is an insignificant value; and thus showed that age and the pain component of the ODI are not related to each other.

The r-value for the correlation result between age and ODI-D is $<0.01$ and the p-value obtained is 0.97 , which is an insignificant value; thus, these results suggest that age and the disability component of the ODI are not related to each other.

The correlation result for the age and FES showed the $\mathrm{r}$-value as -0.05 and the p-value as 0.63 , which is an insignificant value; hence, this suggests that age and FES are not related to each other. Thus the values obtained show that age has no relationship with pain, disability, or fear of falling.

\section{DISCUSSION}

We conducted an experimental study on 100 subjects, whose ages ranged from 40-73 years, to examine the relationship between fear of falling and disability in persons with non-specific LBP. It was found that for the subjects with non-specific LBP there is a moderate correlation between the perceived fear of falling and disability. While at the same time there is a positive and moderately fair correlation between pain and disability, as well as with pain and fear of falling. The LBP disability was estimated by using ODI, and the fear of falling was calculated by the FES. Once fear of falling develops, especially if activities of a subject are limited, it is highly likely to persist, regardless of whether there is fall. The moderate and positive correlation, of around 0.48 between a fear of falling activity and disability in non-specific LBP, highlights the hypothesis that reducing LBP may decrease the risk of falling, as well as disability in patients. Furthermore, individuals, who limit activities because of fear of falling, are at particularly high risk of becoming fallers. Thus, decreasing activity may lead to a decline in function, which leads to a higher risk of subsequent falls. In addition, because individuals who cut back on activities have a higher prevalence of risk factors for falls at baseline, their psychological status alone puts them at a higher risk of becoming fallers [5].

Thus, both disability and fear of falling are related to each other. In this study, the findings that LBP is associated with the subsequent development of severe mobility disability may have important public health implications for a rapidly aging population. Thus, pain results in more and more disability in performing a work, and thus mobility disability further leads to an increased likelihood for further dependence in activities of daily living $[8,9]$.

Another finding of this study is that the LBP is related to a fall, this may be due to the fear of pain, which leads to either frequent falls or fear of falling. Thus, it is clear that the LBP fear of falling and disability are related to each other. Another possible reason may be that LBP leads to a disability to perform any work normally, and at the same time a fear of pain generates a fear of falling and increases the rate of falling for the subjects. At the same time a disability and fear of falling are also linked to each other, as fear of falling leads a person to a decrease in mobility and thus increases the disability further to a greater extent; whereas the converse may also be true, that is, a disability in LBP increases the extent of fear of falling for an individual [2].

In summary, the fear of falling and disability are serious, but potentially preventable conditions, in LBP. Because each is a risk factor for the other, an individual who has one of these factors is at risk for developing the other. This in turn may set off a 'vicious cycle' of fear of falling, and many adverse outcomes can thereby ensue, such as a decline in function or a decrease in quality of life. Further studies are needed to determine whether the primary prevention efforts based on the risk factors for developing disability and fear of falling, as well as the secondary prevention efforts for Fear of falling and disability, can be useful in reducing these adverse outcomes.

There are some limitations present in this study. In this study we did not define a 'fall' when conducting the 
examination. Also, we did not ask how the sample group defined a fall. Other fall-related psychological instruments exist in addition to the FES, which is 'the most widely used' questionnaire, and has been used in the present study; hence there is a possibility that some important points for the evaluation of fear of falling might be missing from the FES questionnaire. Anthropometric data were not taken, such as weight, height, BMI, and previous fall history.

In conclusion, there are three major conclusions from these data. First, low back pain increases the fear of falling. Second, LBP can result in a person becoming disabled. Third, the fear of falling and disability are correlated with each other. More research and more efforts are still needed, such as studies that will evaluate the interventions that may reduce the fear of falling.

\section{CONFLICT OF INTEREST}

No potential conflict of interest relevant to this article was reported.

\section{REFERENCES}

1. Lin CW, Haas M, Maher CG, Machado LA, van Tulder MW. Cost-effectiveness of guideline-endorsed treatments for low back pain: a systematic review. Eur Spine J 2011;20:1024-38.

2. Champagne A, Prince F, Bouffard V, Lafond D. Balance, falls-related self-efficacy, and psychological factors amongst older women with chronic low back pain: a preliminary case-control study. Rehabil Res Pract 2012;2012:430374.

3. Karaman H, Tufek A, Kavak GO, Kaya S, Yildirim ZB, Uysal E, et al. 6-month results of TransDiscal Biacuplasty on patients with discogenic low back pain: preliminary findings. Int J Med Sci 2010;8:1-8.

4. Kwok BC, Mamun K, Chandran M, Wong CH. Evaluation of the Frails' Fall Efficacy by Comparing Treatments (EFFECT) on reducing fall and fear of fall in moderately frail older adults: study protocol for a randomised control trial. Trials 2011;12:155.

5. Swanenburg J, de Bruin ED, Favero K, Uebelhart D, Mulder T. The reliability of postural balance measures in single and dual tasking in elderly fallers and nonfallers. BMC Musculoskelet Disord 2008;9:162.

6. Tinetti ME, Richman D, Powell L. Falls efficacy as a measure of fear of falling. J Gerontol 1990;45:P239-43.

7. Fairbank JC, Pynsent PB. The Oswestry Disability Index. Spine (Phila Pa 1976) 2000;25:2940-52.

8. Mendes da Costa E, Pepersack T, Godin I, Bantuelle M, Petit B, Leveque A. Fear of falling and associated activity restriction in older people. results of a crosssectional study conducted in a Belgian town. Arch Public Health 2012;70:1.

9. Leeuw M, Goossens ME, Linton SJ, Crombez G, Boersma K, Vlaeyen JW. The fear-avoidance model of musculoskeletal pain: current state of scientific evidence. J Behav Med 2007;30:77-94. 